\title{
Novos registros de Surirella Turpin (Bacillariophyta, Surirellaceae) para o Rio Grande do Sul e Brasil
}

\author{
Lezilda Carvalho Torgan ${ }^{1,2}$ e Andrea Saldanha Weber ${ }^{1}$
}

Recebido em 16/05/2006. Aceito em 25/06/2007

\begin{abstract}
RESUMO - (Novos registros de Surirella Turpin (Bacillariophyta, Surirellaceae) para o Rio Grande do Sul e Brasil). O estudo do gênero Surirella em ambientes aquáticos na Planície Costeira do Rio Grande do Sul revelou a presença de S. minuta Brébisson var. peduliformis Frenguelli, S. rumrichorum Metzeltin \& Lange-Bertalot e Surilella chilensis Janisch var. tumida Hustedt. Estas espécies possuem distribuição geográfica restrita à América do Sul. Descrições, ilustrações e comentários sobre as condições ambientais e a distribuição geográfica das referidas espécies são apresentadas.
\end{abstract}

Palavras-chave: diatomáceas, Surirella, taxonomia, distribuição geográfica

\begin{abstract}
New reports of Surirella Turpin (Bacillariophyta, Surirellaceae) for Rio Grande do Sul State and Brazil). The study of Surirella genus in aquatic environments of the Coastal Plain revealed the presence of S. minuta Brébisson var. peduliformis Frenguelli, S. rumrichorum Metzeltin \& Lange-Bertalot and Surirella chilensis Janisch var. tumida Hustedt. The geographic distribution of these species is restricted to South America. Descriptions, illustrations and comments on environmental conditions and geographic distribution of the species are presented.
\end{abstract}

Key words: diatoms, Surirella, taxonomy, geographic distribution

\section{Introdução}

Surirella Turpin é um gênero bentônico podendo, no entanto, ser encontrado freqüentemente no plâncton. Ocorre em diversos ambientes aquáticos, quais sejam, água doce, salobro e marinho o que provavelmente explica, segundo Ruck \& Kociolek (2004) a sua origem polifilética. O grupo das espécies dulcícolas possui valvas iso a heteropolares, um sistema de rafe elevado em quilha que ocupa toda a circunferência valvar e canais alares, que se estendem mais ou menos pronunciadamente em direção à área axial (Round et al. 1990).

Trata-se de um gênero relativamente pouco conhecido no Brasil. Os estudos taxonômicos em águas continentais encontram-se concentrados na região sul do país. No Rio Grande do Sul foram registrados, até o momento, 45 táxons específicos e infra-específicos, predominantemente para rios e lagoas costeiras, onde os estudos foram mais intensamente realizados (Torgan et al. 1999).
No Estado do Paraná, foram identificados um total de 36 táxons infragenéricos, dos quais 19 foram referenciados para barragens, lagoas e tanques (Shirata 1985) e para o plâncton e perifíton de lagos artificiais (Ludwig et al. 2005), sendo que os demais encontramse citados em dissertações não publicadas. Para o Estado de Santa Catarina, são referidos 19 táxons exclusivamente em ambientes lóticos (Fernandes et al. 1990; Rodrigues 1984; Felício-Fernandes 1996; Burliga et al. 2005). Para São Paulo são mencionados cinco táxons (Magrin \& Senna 1997; Magrin \& Senna 2000) e para o Rio de Janeiro, 16 táxons encontrados no plâncton e perifíton de córregos e lagoas costeiras (Menezes \& Dias 2001). Para o Estado de Goiás são mencionados oito táxons (Nogueira et al. dados não publicados), enquanto que, para o Estado do Pará e Distrito Federal apenas dois e um táxon, respectivamente (Moreira Filho et al. 1974; Senna et al. 1998).

Este trabalho tem por objetivo registrar três espécies de Surirella pela primeira vez na Planície

\footnotetext{
Fundação Zoobotânica do Rio Grande do Sul, Museu de Ciências Naturais, C. Postal 1188, 90001-970 Porto Alegre, RS, Brasil

2 Autor para correspondência: lezilda.torgan@fzb.rs.gov.br
} 
Costeira no sul do Brasil. Apresenta suas características morfológicas, métricas, bem como comentários sobre a distribuição e as condições ambientais, onde os táxons foram encontrados, ampliando desta forma o conhecimento taxonômico e distribuição geográfica de representantes deste gênero no país.

\section{Material e métodos}

$\mathrm{O}$ estudo baseou-se em amostragens realizadas em 21 pontos (Tab. 1) distribuídos em lagoas, açudes, banhados e canais, localizados na área da lagoa do Casamento (margem leste) e na área dos Butiazal de Tapes (margem oeste) da laguna dos Patos (30 $10^{\circ}$ '$30^{\circ} 40^{\prime} \mathrm{S}$ e 50³0'-51³0'W), durante os meses de maio e junho (outono) e outubro, novembro e dezembro (primavera) de 2003 (Fig. 1).

As coletas de material planctônico foram obtidas com frascos e com rede (malha de $25 \mu \mathrm{m}$ ) em arraste superficial. As coletas de material perifítico foram efetuadas através de espremido manual de macrófitas aquáticas. Simultaneamente foram efetuadas medições pontuais de temperatura, condutividade, salinidade e pH através de aparelhos marca HACH modelos 50150 e 50050. A preparação das amostras consistiu na oxidação do material com água oxigenada e dicromato de potássio, a uma temperatura de $100{ }^{\circ} \mathrm{C}$, durante uma hora; seguida de consecutivas lavagens, com água destilada. Após cada lavagem, o material foi centrifugado durante cinco minutos a uma velocidade de $2.500 \mathrm{rpm}$. As lâminas foram montadas com Naphrax (IR 1,74). Observou-se ao microscópio óptico (MO) um total de 165 amostras abragendo as zonas litorânea (marginal) e pelágica (central) dos corpos d'água e os dois períodos de amostragem. Registrouse, em fotomicrografias, as variações morfológicas e métricas dos indivíduos encontrados nas lâminas permanentes. Observou-se também material não oxidado para confirmar a presença de plastídios. O material estudado encontra-se tombado no Herbário Prof. Dr. Alarich Schultz (HAS) no Museu de Ciências Naturais da Fundação Zoobotânica do Rio Grande do Sul. O enquadramento taxonômico das espécies foi baseado no sistema de Round et al. (1990).

\section{Resultados e discussão}

A seguir encontram-se as descrições, comentários e ilustrações dos táxons identificados.

Tabela 1. Relação dos pontos, áreas ( $\mathrm{LC}=$ lagoa do Casamento, $\mathrm{BT}=$ Butiazal de Tapes), ambientes e zonas ( $\mathrm{L}=$ litorânea, $\mathrm{P}=$ pelágica), amostrados com suas respectivas coordenadas geográficas.

\begin{tabular}{|c|c|c|c|c|}
\hline Pontos & Áreas & Ambientes & Zonas & Coordenadas geográficas \\
\hline $\mathrm{P} 1$ & $\mathrm{LC}$ & banhado entre L. Capivari e Casamento & $\mathrm{L}$ & $-30^{\circ} 14^{\prime} 29^{\prime \prime} /-50^{\circ} 33^{\prime} 55^{\prime \prime}$ \\
\hline $\mathrm{P} 2$ & $\mathrm{LC}$ & banhado entre L. Capivari e Casamento & $\mathrm{L}$ & $-30^{\circ} 14^{\prime} 32^{\prime \prime} /-50^{\circ} 33^{\prime} 59^{\prime \prime}$ \\
\hline P3 & $\mathrm{LC}$ & lagoa do Capivari & $\mathrm{P}$ & $-30^{\circ} 14^{\prime} 38^{\prime \prime} /-50^{\circ} 33^{\prime} 15^{\prime \prime}$ \\
\hline $\mathrm{P} 4$ & $\mathrm{LC}$ & lagoa do Casamento & $\mathrm{P}$ & $-30^{\circ} 16^{\prime} 40^{\prime \prime} /-50^{\circ} 33^{\prime} 25^{\prime \prime}$ \\
\hline P5 & $\mathrm{LC}$ & banhado Fazenda Rincão Anastácio & $\mathrm{L}$ & $-30^{\circ} 22^{\prime} 26^{\prime \prime} /-50^{\circ} 40^{\prime} 48^{\prime \prime}$ \\
\hline P6 & $\mathrm{LC}$ & banhado Fazenda Rincão Anastácio & $\mathrm{L}$ & $-30^{\circ} 22^{\prime} 26^{\prime \prime} /-50^{\circ} 40^{\prime} 48^{\prime \prime}$ \\
\hline P7 & $\mathrm{LC}$ & lagoa dos Gateados Norte & $\mathrm{L}$ & $-30^{\circ} 28^{\prime} 18^{\prime \prime} /-50^{\circ} 40^{\prime} 4 "$ \\
\hline P8 & $\mathrm{LC}$ & lagoa dos Gateados Norte & $\mathrm{L}$ & $-30^{\circ} 30^{\prime} 19^{\prime \prime} /-5039^{\prime} 50 ”$ \\
\hline P9A & $\mathrm{LC}$ & lagoa dos Gateados Norte & $\mathrm{P}$ & $-30^{\circ} 30^{\prime} 26^{\prime \prime} /-50^{\circ} 39^{\prime} 12^{\prime \prime}$ \\
\hline $\mathrm{P} 9 \mathrm{~B}$ & $\mathrm{LC}$ & lagoa dos Gateados Norte & $\mathrm{P}$ & $-30^{\circ} 30^{\prime} 39^{\prime \prime} / /-50^{\circ} 39^{\prime} 49^{\prime \prime}$ \\
\hline $\mathrm{P} 10$ & $\mathrm{LC}$ & canal do Sangradouro & $\mathrm{L}$ & $-30^{\circ} 26^{\prime} 60^{\prime \prime} /-50^{\circ} 39^{\prime} 29^{\prime \prime}$ \\
\hline P11 & $\mathrm{LC}$ & lagoa dos Gateados Sul & $\mathrm{L}$ & $-30^{\circ} 32^{\prime} 8^{\prime \prime} /-50^{\circ} 39^{\prime} 39^{\prime \prime}$ \\
\hline $\mathrm{P} 12 \mathrm{~A}$ & $\mathrm{LC}$ & lagoa dos Gateados Sul & $\mathrm{P}$ & $-30^{\circ} 31^{\prime} 34^{\prime \prime} /-50^{\circ} 39^{\prime} 33^{\prime \prime}$ \\
\hline $\mathrm{P} 12 \mathrm{~B}$ & $\mathrm{LC}$ & lagoa dos Gateados Sul & $\mathrm{P}$ & $-30^{\circ} 31^{\prime} 29^{\prime \prime} /-50^{\circ} 38^{\prime} 37^{\prime \prime}$ \\
\hline P13 & BT & lagoa Charutão & $\mathrm{L}$ & $-30^{\circ} 30^{\prime} 58^{\prime \prime} /-51^{\circ} 21^{\prime} 17^{\prime \prime}$ \\
\hline $\mathrm{P} 14$ & $\mathrm{BT}$ & lagoa Charutão & $\mathrm{P}$ & $-30^{\circ} 31^{\prime} 14^{\prime \prime} /-51^{\circ} 21^{\prime} 25^{\prime \prime}$ \\
\hline P15 & $\mathrm{BT}$ & açude Fazenda São Miguel & $\mathrm{L}$ & $-30^{\circ} 31^{\prime} 7^{\prime \prime} /-51^{\circ} 22^{\prime} 23^{\prime \prime}$ \\
\hline P16 & $\mathrm{BT}$ & lagoa das Capivaras & $\mathrm{L}$ & $-30^{\circ} 28^{\prime} 11^{\prime \prime} /-51^{\circ} 16^{\prime} 30^{\prime \prime}$ \\
\hline P17 & BT & lagoa das Capivaras & $\mathrm{P}$ & $-30^{\circ} 28^{\prime} 11^{\prime \prime} /-51^{\circ} 16^{\prime} 30^{\prime \prime}$ \\
\hline P18 & BT & banhado com Sphagnum & $\mathrm{L}$ & $-30^{\circ} 28^{\prime} 24^{\prime \prime} /-51^{\circ} 16^{\prime} 38^{\prime \prime}$ \\
\hline P19 & BT & lagoinha entre dunas & $\mathrm{L}$ & $-30^{\circ} 28^{\prime} 25^{\prime \prime} /-51^{\circ} 16^{\prime} 36^{\prime \prime}$ \\
\hline $\mathrm{P} 20$ & BT & banhado entre dunas & $\mathrm{L}$ & $-30^{\circ} 28^{\prime} 20^{\prime \prime} /-51^{\circ} 16^{\prime} 30^{\prime \prime}$ \\
\hline $\mathrm{P} 21$ & BT & lagoa Redonda & $\mathrm{L} / \mathrm{P}$ & $-30^{\circ} 31^{\prime} 36^{\prime \prime} /-51^{\circ} 21^{\prime} 34^{\prime \prime}$ \\
\hline
\end{tabular}




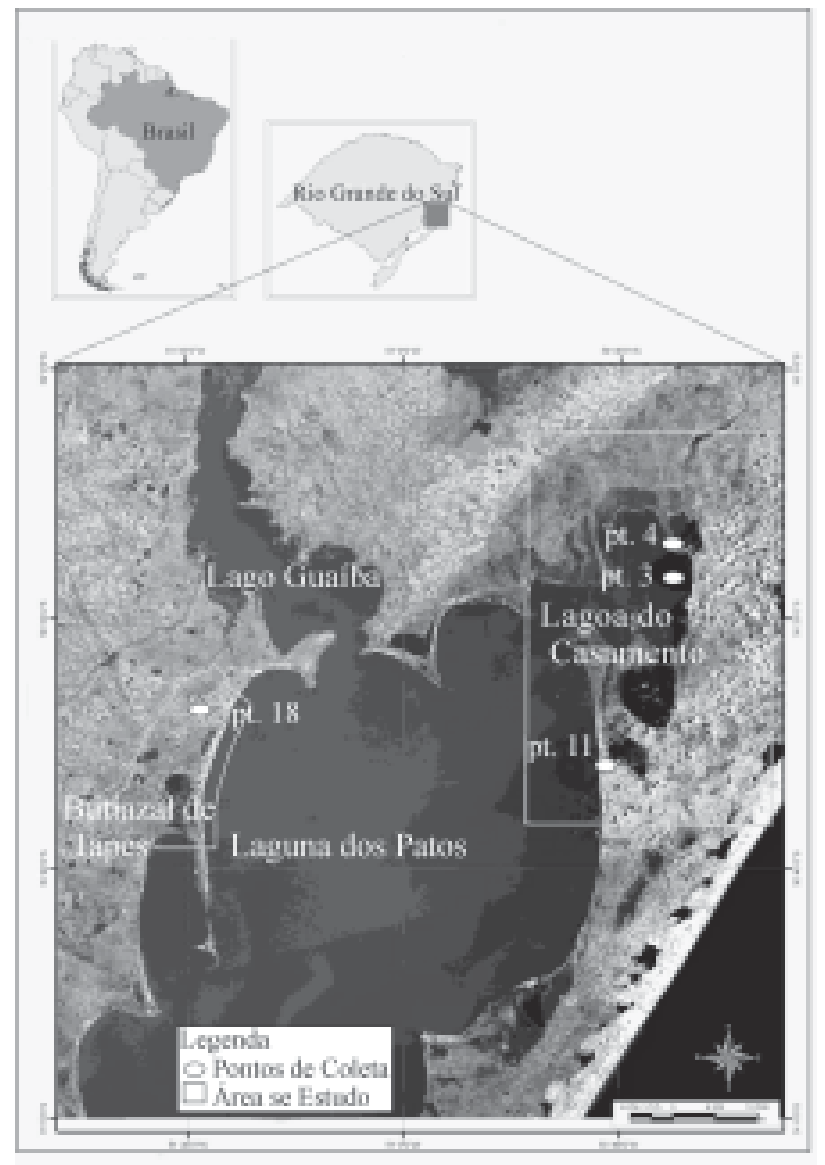

Figura 1. Localização dos pontos de amostragem na área de estudo RS, Brasil: lagoa do Casamento (pt. 4), lagoa do Capivari (pt. 3), lagoa dos Gateados Sul (pt. 11) e banhado de Sphagnum (pt. 18).

\section{BACILLARIOPHYTA}

\section{BACILLARIOPHYCEAE}

\section{SURIRELLALES}

\section{SURIRELLACEAE}

Surirella chilensis Janisch var. tumida Hustedt, 1927 Fig. 2, 3

Valvas lanceoladas, isopolares, com pólos cuneado-arredondados. Área axial elíptica não alcançando os pólos. Canais alares estendendo-se até a área axial. Medidas: comprimento 97,5-112 $\mu \mathrm{m}$; largura 54-60 $\mu \mathrm{m}$; canais alares 20-26 em $100 \mu \mathrm{m}$.

Material examinado: BRASIL. Rio Grande do Sul: Capivari do Sul, lagoa do Capivari, pt. 3, zona pelágica, 18/XI/2003, Cardoso s.n. (HAS 104343, lâm. n. 5842, 5843); Palmares do Sul, lagoa do Casamento, pt. 4, zona pelágica, 18/XI/2003, Cardoso s.n. (HAS 104352, lâm. n. 5847).
Ocorrência: primeiro registro para o Brasil.

Esta variedade foi registrada, até o momento, somente para a região do Chile, no deserto de Atacama entre 4.000 e $4.300 \mathrm{~m}$ de altitude, inicialmente em sedimentos superficiais na zona litorânea da bacia do rio Loa (Hustedt 1927) e na bacia do rio Lauca (Rumrich et al. 2000) e, posteriormente, na lagoa salina de Punta Negra (Diaz \& Maidana 2005).

$\mathrm{Na}$ área de estudo, este táxon restringiu-se a duas lagoas com pH 6,9 , temperatura média de $22,4{ }^{\circ} \mathrm{C}$, condutividade de 80 e $119 \mu \mathrm{S} \mathrm{cm}$ c $^{-1}$ e salinidade não detectável, na estação de primavera. É importante mencionar que $S$. chilensis var. tumida encontravase restrita a ambientes de altas altitudes em águas alcalinas $(\mathrm{pH} \mathrm{8,2)} \mathrm{e} \mathrm{salobras} \mathrm{(salinidade} \mathrm{superior} \mathrm{a}$ $10 \mathrm{~g} \mathrm{~L}^{-1}$ ) diferindo das condições por nós observadas. Uma hipótese para explicar a presença desta variedade na Planície Costeira do Rio Grande do Sul é a possibilidade do material ter sido introduzido através de aves aquáticas que possuem uma rota no sistema migratório austral da América do Sul (Sick 1983). A sobrevivência e transporte de diatomáceas, seja nas penas de aves (Croll \& Holmes 1982) ou em seu trato digestivo (Proctor 1959) são registrados na literatura, demonstrando a importância desses organismos na dispersão de microalgas.

\section{Surirella minuta Brébisson var. peduliformis \\ Frenguelli, 1941}

Fig. 4

Valvas lineares, heteropolares, com pólo superior amplamente arredondado e pólo inferior cuneadoarredondado. Área axial indiferenciada. Canais alares restritos à margem valvar. Estrias transversais tênues. Medidas: comprimento 31-53 $\mu \mathrm{m}$; largura máxima 9-10 $\mu \mathrm{m}$; largura mínima 8-9 $\mu \mathrm{m}$; canais alares $63 \mathrm{em}$ $100 \mu \mathrm{m}$ e estrias $30 \mathrm{em} 10 \mu \mathrm{m}$.

Material examinado: BRASIL. Rio Grande do Sul: Mostardas, lagoa dos Gateados Sul, pt. 11, zona pelágica, 9/V/2003, Werner s.n. (HAS 104174, lâm. n. 5807).

Ocorrência: primeiro registro para o Estado.

Esta variedade encontra-se restrita à região subtropical da América do Sul. Foi referenciada pela primeira vez no plâncton do estuário do rio de La Plata e em charcos pantanosos (Frenguelli 1941), sendo citada posteriormente por Metzeltin \& GarcíaRodríguez (2003) e Metzeltin et al. (2005) para o rio de La Plata.

No Brasil, este táxon foi referenciado por Ferrari (dados não publicados) como S. minuta var. minuta, para os rios da bacia hidrográfica do Ivaí, 
Prudentópolis, Paraná e em um lago do Jardim Botânico de Curitiba (Ludwig et al. 2005).

Pouco se conhece sobre as condições ambientais em que esta variedade ocorre. Os únicos dados disponíveis são os Frenguelli (1941) que menciona sua presença em águas com pH 7,6.

Este táxon foi raro e de ocorrência restrita, porque observou-se apenas dois indivíduos somente na lagoa dos Gateados Sul com temperatura de $17^{\circ} \mathrm{C}, \mathrm{pH}$ 6,8 e alta condutividade $\left(187 \mu \mathrm{S} \mathrm{cm}^{-1}\right)$, na estação de outono.

Surirella rumrichorum Metzeltin \& Lange-Bertalot, 1998

Fig. 5-8

Valvas lineares, isopolares, constritas na região central, com pólos cuneado-arredondados. Área axial
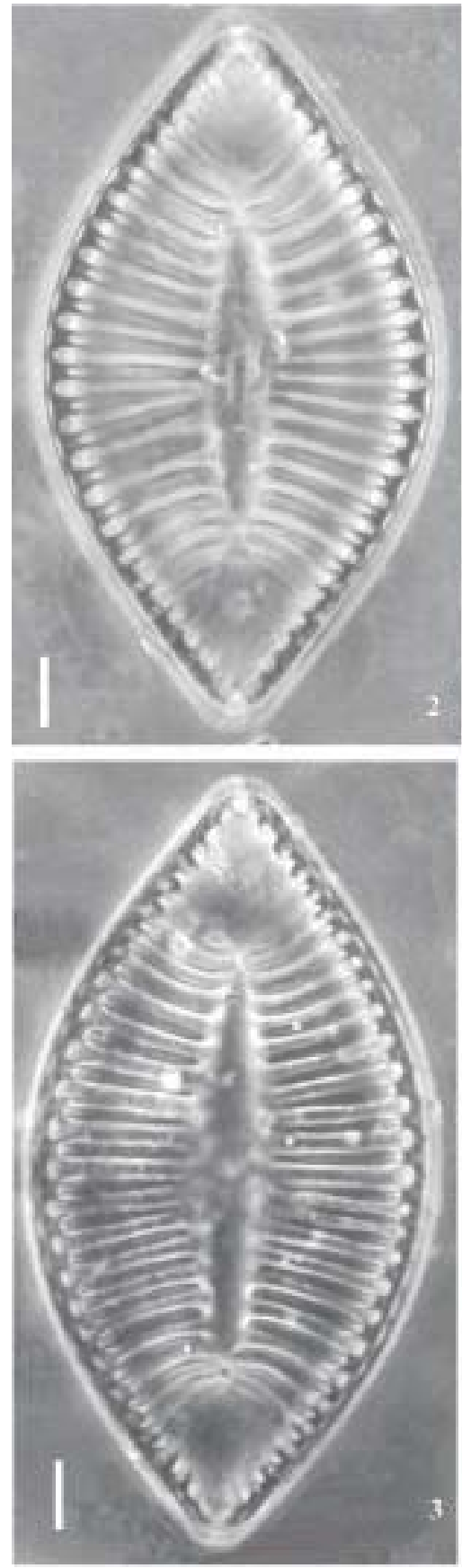
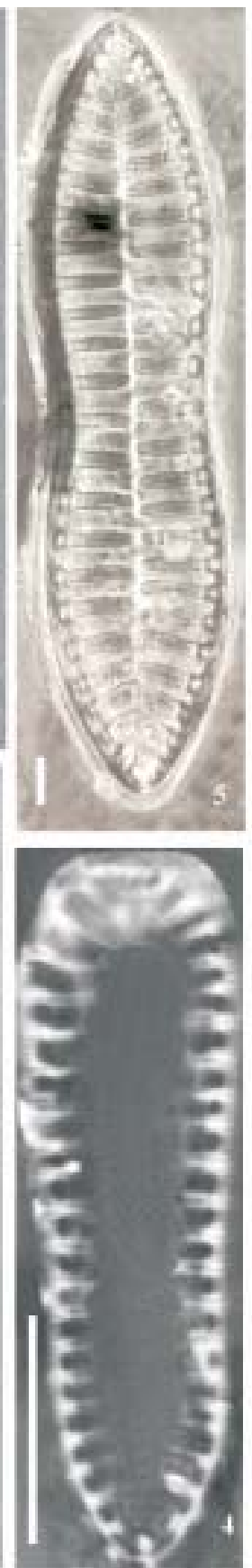
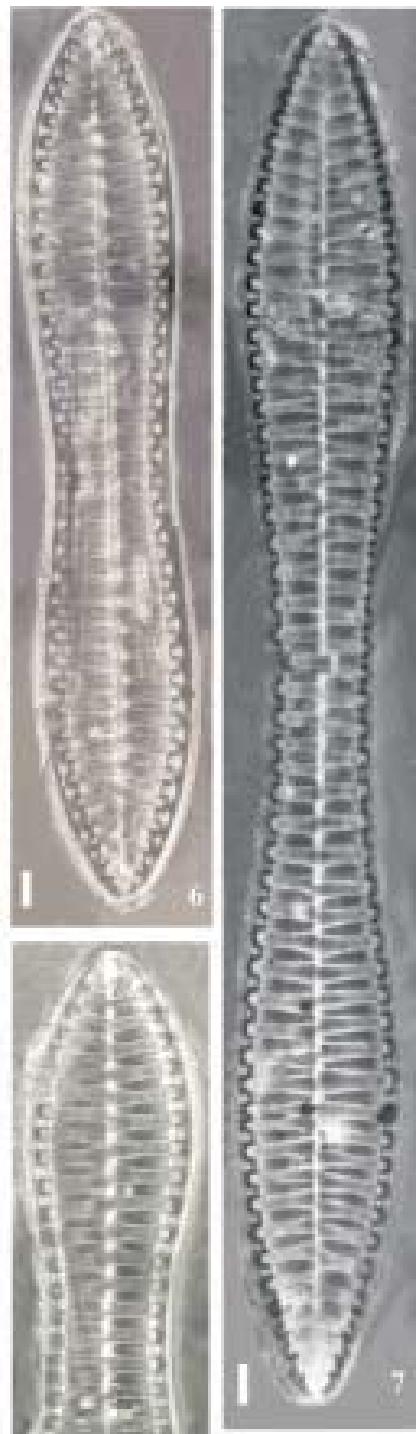

Figuras 2-8. Representação das espécies em vista valvar. 2-3. Surirella chilensis Janisch var. tumida Hustedt. 4. Surirella minuta Brébisson var. peduliformis Frenguelli. 5-8. Surirella rumrichorum Metzeltin \& Lange-Bertalot. Escala: $10 \mu \mathrm{m}$. 
linear estreita. Canais alares estendendo-se até a área axial. Medidas: comprimento 161-335 $\mu \mathrm{m}$; largura máxima 34-41 $\mu \mathrm{m}$; largura mínima 25-32,5 $\mu \mathrm{m}$ e canais alares 14-16 em $100 \mu \mathrm{m}$.

Material examinado: BRASIL. Rio Grande do Sul: Barra do Ribeiro, banhado com Sphagnum, pt. 18, zona litorânea, 4/VI/2003, Torgan \& Werner s.n. (HAS 104221, lâm. n. 5831, 5832); 3/XII/2003, Cardoso s.n. (HAS 104440, lâm. n. 5869).

Ocorrência: primeiro registro para o Estado.

Esta espécie foi identificada por Metzeltin \& Lange-Bertalot (1998) com base em material oriundo da laguna Negra, Venezuela e posteriormente registrada para o lago Mucubaji neste mesmo país Ruck \& Kociolek (2004). No Brasil esta espécie foi pela primeira vez encontrada em rios e córregos na Reserva Biológica de Poço das Antas, Rio de Janeiro, por Costa (1995) e identificada como S. thienemanni Hustedt. A mesma, entretanto, difere de S. rumrichorum por apresentar área axial linearelíptica não alcançando os pólos, menores dimensões

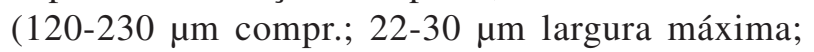
18-23 $\mu \mathrm{m}$ larg. mínima) e maior número de canais alares (20-24 em $100 \mu \mathrm{m})$.

Vários indivíduos $(\mathrm{n}=8)$ da população estudada possuem menores dimensões em relação ao material encontrado na Venezuela (280-400 $\mu \mathrm{m}$ compr. e 40-52 $\mu \mathrm{m}$ larg. máxima) assemelhando-se mais aos exemplares do Rio de Janeiro (235-397 $\mu \mathrm{m}$ compr. e 35-36 $\mu \mathrm{m}$ larg. máxima). Com referência ao número de canais alares, valores intermediários entre (12-14 em $100 \mu \mathrm{m})$ e $(17-18 \mathrm{em} 100 \mu \mathrm{m})$ mencionados para os locais acima referidos, respectivamente, foram observados.

Com relação à distribuição na área de estudo, esta espécie esteve restrita a um banhado coberto com Sphagnum, onde o $\mathrm{pH}$ variou entre 4,3 e 6,4 e a temperatura da água entre $21^{\circ} \mathrm{Ce} 32,5^{\circ} \mathrm{C}$. Entretanto, não podemos afirmar que essas condições sejam favoráveis ao desenvolvimento dessa espécie, pois apesar de terem sido encontrados em grande número nas amostras, os indivíduos não apresentaram plastídios.

Pode-se concluir que Surirella chilensis var. tumida, S. minuta var. peduliformis e S. rumrichorum possuem distribuição geográfica restrita, tratando-se possivelmente de espécies endêmicas da América do Sul, pelo fato de, até o momento, terem sido registradas somente para o Chile, Venezuela, Argentina e Brasil, e que $S$. chilensis var. tumida é um táxon que pode ser encontrado tanto em ambientes situados em grandes altitudes quanto na Planície Costeira, ao nível do mar.

\section{Agradecimentos}

À equipe de pesquisadores e funcionários do Museu de Ciências Naturais/Fundação Zoobotânica do Rio Grande do Sul, ao ornitólogo Dr. Glayson Bencke, à geógrafa Arlete Pasqualetto e ao acadêmico Everton Luís Luz de Quadros, do Centro de Geoprocessamento do MCN/FZB, pelo auxílio prestado; ao Conselho Nacional de Ciência e Tecnologia - CNPq, pelas bolsas de Produtividade em Pesquisa e de Iniciação Científica concedidas à primeira e segunda autora, respectivamente.

\section{Referências bibliográficas}

Burliga, A.L.; Torgan, L.C.; Nobrega, E.A; Beaumord, A.C.; Costa, C.O. \& Yamauti, D.V. 2005. Diatomáceas epilíticas do rio Itajaí-Mirim, Santa Catarina, Brasil. Acta Scientarum, Biological Sciences 27: 415-421.

Costa, J.C.F. 1995. Diatomáceas (Bacillariophyta) da Reserva Biológica de Poço das Antas, município de Silva Jardim, Rio de Janeiro, Brasil. Iheringia, Série Botânica 46: 57-143.

Croll, D.A. \& Holmes, R.W. 1982. A Note on the Occurrence of Diatoms on the Feathers of diving Seabirds. The Auk 99: 765-766.

Díaz, C. \& Maidana, N. 2005. Diatomeas de los Salares Atacama y Punta Negra. II Región, Chile. Chile, Centro de Ecología Aplicada.

Fernandes, L.F.; Souza-Mosimann, R.M. \& FelícioFernandes, G. 1990. Diatomáceas (Bacillariophyceae) do Rio Ratones, Florianópolis, Santa Catarina, Brasil: I Baixo Curso e Estuário. Ínsula 20: 11-112.

Felício-Fernandes, G. 1996. Diatomáceas do Rio Tavares, Manguezal do Rio Tavares, Florianópolis, Santa Catarina, Brasil. II. Penatae. Ínsula 25: 69-192.

Frenguelli, J. 1941. Diatomeas del Río de La Plata, Argentina. Revista del Museo de La Plata, Sección Botánica 15: 213-334.

Hustedt, F. 1927. Fossile Bacillariaceen aus dem Loa-Becken in der Atacama-Wüste, Chile. Archiv für Hydrobiologie und Planktokunde 18: 224-251.

Ludwig, T.A.V.; Bigunas, P.I.T.; Neiva, T.F.; Coquemala, V. \& Piccinini, C. 2005. Diatomáceas (Ochorophyta) dos Lagos do Jardim Botânico, Curitiba, Paraná. Pp. 301-323. In: Anais da X Reunião Brasileira de Ficologia. Rio de Janeiro, Sociedade Brasileira de Ficologia.

Magrin, A.G.E. \& Senna, P.A.C. 1997. Composição e Dinâmica de Diatomáceas Planctônicas em uma Lagoa da Planície de Inundação do médio Mogi-Guaçu, estado de São Paulo, Brasil. Pp. 247-276. In: Anais do VIII Seminário Regional de Ecologia. São Paulo.

Magrin, A.G.E. \& Senna, P.A.C. 2000. Diatomáceas (Bacillariophyta) da Lagoa do Diogo e seus trechos fluviais: Córrego Cafundó e Rio Mogi-Guaçu. 2. Classe Bacillariophyceae. Pp. 415-430. In: J.S.R. Pires \& J.E. Santos (orgs.). Estudos Integrados em Ecossistemas Estação Ecológica de Jataí. São Carlos. 
Menezes, M. \& Dias, I.C.A. 2001. Biodiversidade de Algas de Ambientes Continentais do Estado do Rio de Janeiro. Rio de Janeiro, Museu Nacional.

Metzeltin, D. \& Lange-Bertalot, H. 1998. Tropische Diatomeen in Südamerika I. Ruggell, A.R.G Verlag. (Iconographia Diatomologica, v. 5).

Metzeltin, M. \& García-Rodrigues, F. 2003. Las Diatomeas Uruguayas. Montevideo, DI.R.A.C.

Rodrigues, L. 1984. Contribuição ao conhecimento das Diatomáceas do Rio Tubarão, Santa Catarina, Brasil. Ínsula 14: 47-120.

Metzeltin, D., Lange-Bertalot, H. \& García-Rodríguez, F. 2005. Diatoms of Uruguay. Rugell, A.R.G. Verlag. (Iconographia Diatomologica, v. 15).

Moreira Filho, H.; Moreira, I.M.V. \& Cecy, I.I.T. 1974. Diatomáceas do Rio Guama (Foz do rio - Belém - Estado do Pará). Leandra 4-5: 123-135.

Proctor, V.W. 1959. Dispersal of Fresh-Water Algae by Migratory Water Birds. Science 130: 623-624.

Round, F.E; Crawford, R.M. \& Mann, D.G. 1990. The Diatoms - Biology \& morphology of the genera. Cambridge, Cambridge University Press.
Ruck, E.C. \& Kociolek, J.P. 2004. Preliminary Phylogeny of the Family Surirellaceae (Bacillariophyta). Berlim, J. Cramer. (Bibliotheca Diatomologica, v. 50).

Rumrich, U.; Lange-Bertalot, H. \& Rumrich, M. 2000. Diatomeen der Anden von Venezuela bis Patagonien / Tierra del Fuego. Ruggell, A.R.G Verlag. (Iconographia Diatomologica, v. 9).

Senna, P.A.C.; Souza, M.G.M. \& Compère, P. 1998. A check-list of the algae of the Federal District (Brazil), Wetteren, Universa. (Scripta Botanica Belgica, v. 16).

Shirata, M.T. 1985. Catálogo de Diatomáceas (Crysophyta, Bacillariophyceae) de água doce do Estado do Paraná, Brasil. Estudos de Biologia 12: 3-63.

Sick, H. 1983. Migrações de Aves na América do Sul Continental. Brasília, CEMAVE. (Publicação Técnica, 2).

Torgan, L.C.; Becker, V. \& Prates, H.M. 1999. Checklist das diatomáceas (Bacillariophyceae) de ambientes de águas continentais e costeiros do Estado do Rio Grande do Sul. Iheringia, Série Botânica 52: 89-144. 